\title{
Influence of Mechanical Stress in a Packaged Frequency-Modulated MEMS Accelerometer
}

\author{
Eurico E. Moreira* ${ }^{* \dagger}$, Burkhard Kuhlmann*, Filipe S. Alves ${ }^{\dagger}$, Rosana A. Dias ${ }^{\dagger}$, Jorge Cabral ${ }^{*}$, João Gaspar ${ }^{\dagger}$ and Luis A. Rocha ${ }^{*}$ \\ *Algortmi Center, University of Minho, Guimarães, Portugal. \\ †International Iberian Nanotechnology Laboratory (INL), Braga, Portugal. \\ ॠAutomotive Electronics group, Robert Bosch GmbH, Reutlingen, Germany.
}

\begin{abstract}
Frequency modulated accelerometers composed of two double-ended tuning fork (DETF) resonators on a differential configuration were characterized for their sensitivity to force applied to their package. Commonly, differential architectures are employed to cancel commonmode errors, such as the mechanical stress or temperature dependency. The device dependence to mechanical stress was experimentally measured for forces up to $15 \mathrm{~N}$ and a reduction of about 5.6 times was obtained on the differential measurement. Additionally, the silicon dies were glued to chip-carriers using two different glues with distinct properties, and their sensitivity to stress was compared. The effectiveness of a viscoelastic glue over an epoxy-based glue for stress decoupling was tested. Long-term measurements under constant force were experimentally performed and for a time period of approximately $100 \mathrm{~min}$, the stress relaxation and creeping of the viscoelastic glue enabled the recovery to the initial output of the sensor.
\end{abstract}

Keywords-MEMS accelerometer, double-ended tuning fork resonators, mechanical stress.

\section{INTRODUCTION}

Frequency modulated MEMS accelerometers have been investigated due to vacuum encapsulation capabilities [1], high dynamic range and quasi-digital output [2], among other benefits. Low pressure encapsulation has several advantages regarding a higher integrability, for example in inertial measurement units (IMU), since usually, MEMS gyroscopes and magnetometers require high quality factors. Therefore, by operating accelerometers in vacuum, singleprocess and single die multi-axis IMU encapsulated at the same pressure become a possibility [3]. This would result in a simpler and faster fabrication/packaging process and consequently lead to a decrease in cost and size, making new applications possible, mainly for automotive and consumer electronics.

Frequency modulated accelerometers based on different types of resonators for in-plane and out-off-plane measurement have already been presented in the literature, showing the research interest due to the before mentioned advantages. The response of these sensors has been studied for different stimuli and drifts due to common mode errors such as, temperature dependency (Young's modulus change) and thermal stress. Some solutions to minimize these effects were already presented, such as a strategic anchor placement, the use of stress decoupling structures [4], the use of a partially glues sample [5], bonding the devices with soft glues and the integration of heater elements to keep the resonators at a constant temperature [6]. In addition, devices with dummy resonators only for temperature instrumentation [7] and differential architectures were also presented. The research effort on these solutions was focused on increasing the device's bias stability. Bias stability is an important parameter which represents how stable the accelerometer output remains when no external acceleration is applied over a given period. Besides temperature, bias stability can also be deteriorated due to charge effects or flicker noise. Although most of the authors address the influence of these effects on the overall performance of the devices, the mechanical stress introduced by external factors is neglected, although it can yield reliability concerns. This mechanical stress can be induced on the accelerometer by any force applied on the surroundings of the device, for instance, on the printedcircuit board (PCB) were this last is inserted. Here, a study on the influence of stress induced through a force directly applied on the device package was performed, using two samples bonded with different glues (distinct mechanical properties).

\section{DEVICE DESCRIPTION AND FABRICATION}

The accelerometers tested are composed of two doubleended tuning fork resonators (left and right DETF) on a differential configuration. The stiffness of the DETF beams endure a change resulting in a natural frequency shift when a force is applied to the extremities. Ideally, on an accelerometer, this force should be applied exclusively as the result of the inertial-mass displacement, however, stress on the anchor points can also yield a frequency drift, highlighting the need for stress decoupling techniques added to the device or packaging.

To increase the device sensitivity to external accelerations, a force amplification mechanism (levers) was added to the devices (see Fig. 1). The devices shown and the instrumentation implemented for the resonators closed-loop operation are in further detail described in [8], [9].

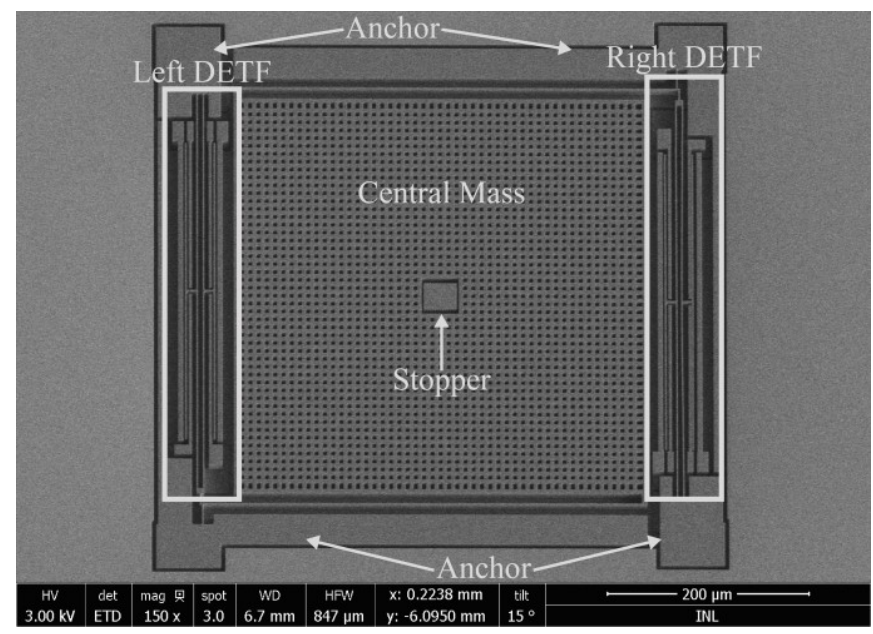

Fig. 1. SEM picture of the frequency modulated accelerometer composed by two DETF on a differential configuration. 
The accelerometers were fabricated in a Bosch standard silicon surface micromachining process and encapsulated in vacuum at $150 \mathrm{~Pa}$. The device design was constrained to the rules and requirements of the fabrication process, to enable sensor integration.

\section{DeVICE PACKAgING AND GLUe Properties}

After the fabrication, the dies containing the accelerometer were glued to ceramic leadless chip carriers (CLCC) with 44 pins and wire bonded. Prototypes bonded using two different glues with distinct properties were assembled to enable the study of their decoupling capabilities.

The glues are based on epoxy (hard-glue) and polydimethylsiloxane (PDMS - soft-glue) materials, having different mechanical properties. While the epoxy-based glue (DELO ${ }^{\circledR}$ MONOPOX) is described by a high Young's modulus and a stable behaviour (no movement of the glue over-time), the PDMS-based glue presents viscoelastic characteristics. PDMS profits from stress-relaxation and creeping, mechanical properties intrinsic to viscoelastic materials. In other words, the material exhibits viscous and elastic behaviour when undergoing a deformation. Depending on the time scale, this material can behave as a solid, a fluid or the combination of both [10].

Stress relaxation explains the relief of induced stress when undergoing a constant strain. This effect deems a spring effect occurring over a long period of time. Stress relaxation occurs in visco-elastic materials because a rearrangement of the material on the molecular or micro-scale level. The re-arrangement is called creep and can be defined as a tendency of a material to move slowly/deform when experiencing a constant stress [10].

The glues' properties available in the technical datasheet are listed in TABLE I. Note, soft-glues are usually used in the semiconductor industry to decouple external stress from devices.

TABLE I. LIST OF GLUE PROPPERTIES USED TO BOND THE PROTOTYPE DIES TO CLLC

\begin{tabular}{|c|c|c|}
\hline Manufacturer & DELO ${ }^{\circledR}$ & Wacker Chemie AG \\
\hline Reference & $\begin{array}{c}\text { MONOPOX } \\
\text { MK096 }\end{array}$ & SEMICOSIL ${ }^{\circledR} 988 / 1 \mathrm{k}$ \\
\hline Base & Epoxy Resin & PDMS \\
\hline Colour & Red & Transparent \\
\hline Density $\left(\mathbf{2 3}^{{ }^{\circ}} \mathbf{C}\right)$ & $1.1 \mathrm{~g} / \mathrm{cm}^{3}$ & $1.1 \mathrm{~g} / \mathrm{cm}^{3}$ \\
\hline Tensile Strength & $60 \mathrm{MPa}$ & $4.5 \mathrm{MPa}$ \\
\hline Young's Modulus & $3500 \mathrm{MPa}$ & $\begin{array}{c}0.5 \mathrm{MPa}(\text { at } 100 \% \\
\text { elongation})\end{array}$ \\
\hline Type of cure $^{3}$ & Thermal & Thermal \\
\hline Classified as: & Hard-glue & Soft-glue \\
\hline
\end{tabular}

\section{EXPERIMENTAL RESULTS}

\section{A. Test procedure and experimental setup}

The resonance frequency drift of the resonators composing the accelerometers was measured in a force/displacement setup for forces up to $15 \mathrm{~N}$ and the data of the load-cell and the accelerometer were recorded simultaneously. The force/displacement instrument moves vertically over a PCB containing the device and a tip enables the application of a controlled force over a small area. The PCB was placed on a support platform and only the corners were fixed, to allow the board to bend. The different components of the setup used for these experiments are shown in Fig. 2.

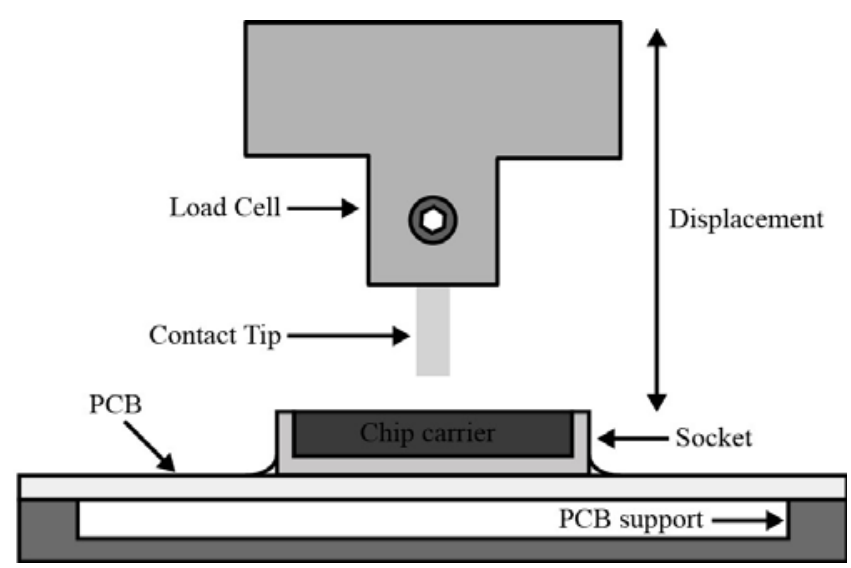

Fig. 2. Schematic of the test setup used to measure a load applied directly to the chip carrier.

To avoid short circuiting the device or damaging the wire-bonding, the devices were tested on the testing point depicted in Fig. 3. The packaging of the device can be observed in the same figure.

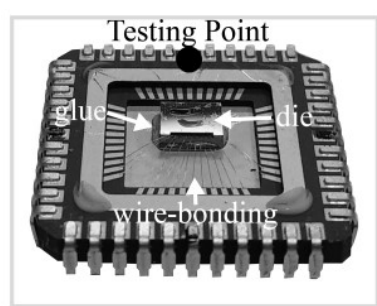

CLCC chip carrier

Fig. 3. Picture of the device packaging and the point where the force was applied by the tip of the force/displacement station.

\section{B. Mechanical Stress dependence}

The frequency shift of the two DETF (single-ended and differential measurement) resulting from a maximum applied force of $15 \mathrm{~N}$ directly on the chip carrier at the testing point of a sample bonded with hard-glue, is shown in Fig. 4. The force was experimentally measured by the load-cell present on the force/displacement station. The DETF are significantly influenced by the external force $(26.2 \mathrm{~Hz} / \mathrm{N}$ for the right DETF) but the differential measurements (left DETF - right DETF) lowered the dependence to $4.67 \mathrm{~Hz} / \mathrm{N}$, yielding a reduction of 5.6 times. The differential measurement does not fully eliminate the drift since the DETF on the left and right have different sensitivities to stress (fabrication mismatches). and/or the resulting stress amplitude is not equal on both resonators

Finite-element method (FEM) analysis were also performed to validate the experimental results (see Fig. 5). A modal analysis of the resonator natural frequency was achieved for a constant force $(5 \mathrm{mN})$ at different angles 


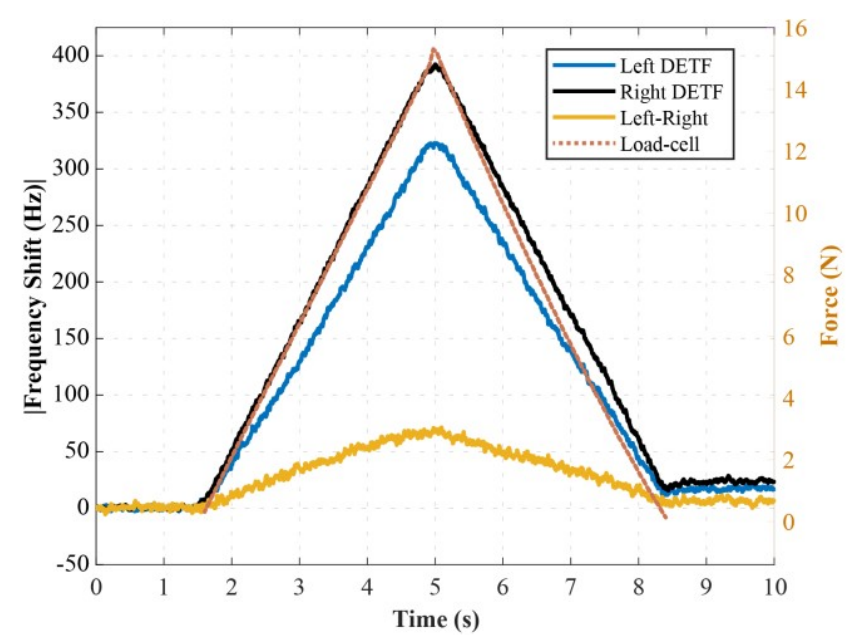

Fig. 4. Experimental results showing the mechanical stress sensitivity with and without the differential measurement for a force applied on the testing point.

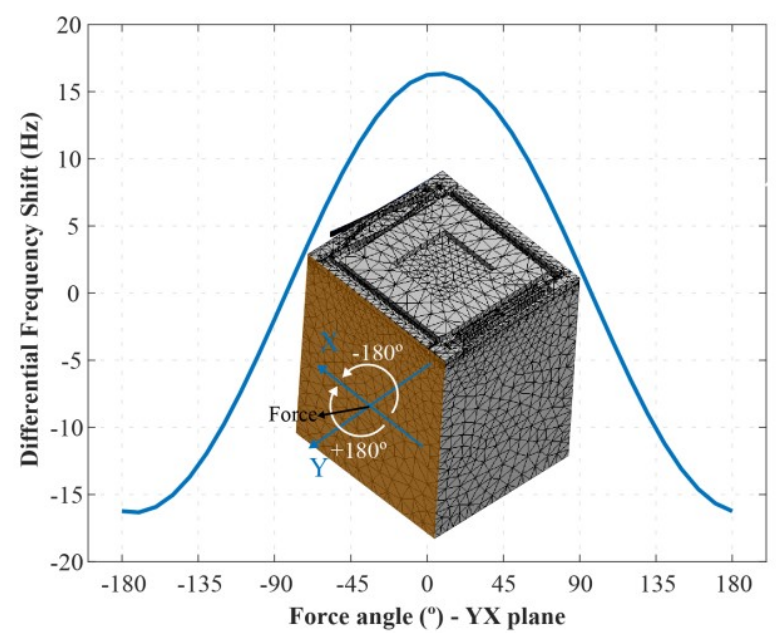

Fig. 5. Simulation of the differential frequency shift for a force $(5 \mathrm{mN})$ applied in the $\mathrm{YX}$ plane at different angles $\left[-180^{\circ}\right.$ to $\left.180^{\circ}\right]$.

applied to one face of the silicon die. From this simulation, the differential frequency shift was extracted, and one can conclude that the frequency drift is dependent on the force angle. The angle influences the stress uniformity changing the DETF response. In other words, the uniformity of the stress on the die directly impacts the effectiveness of the differential architecture, since a good uniformity results in similar drifts on both sides and therefore a full compensation. While a reduced uniformity increases the sensitivity to stress (higher drift mismatch).

The experimental test shown in Fig. 4 was repeated 10 times, to validate the repeatability of the measurements and the testing procedure. In Fig. 6, the frequency drift for the 10 test cycles is shown, showing an average drift of $68.6 \mathrm{~Hz}$ and a standard deviation of $1.2 \mathrm{~Hz}$ for the differential measurement.

\section{Comparison Soft glue/Hard glue}

The same test was repeated, this time using two samples, one mounted on hard-glue and the second on soft glue. The response of the individual DETF together with the differential measurement is shown in Fig. 7. The maximum force applied was $15 \mathrm{~N}$, and for this load, maximum drifts of

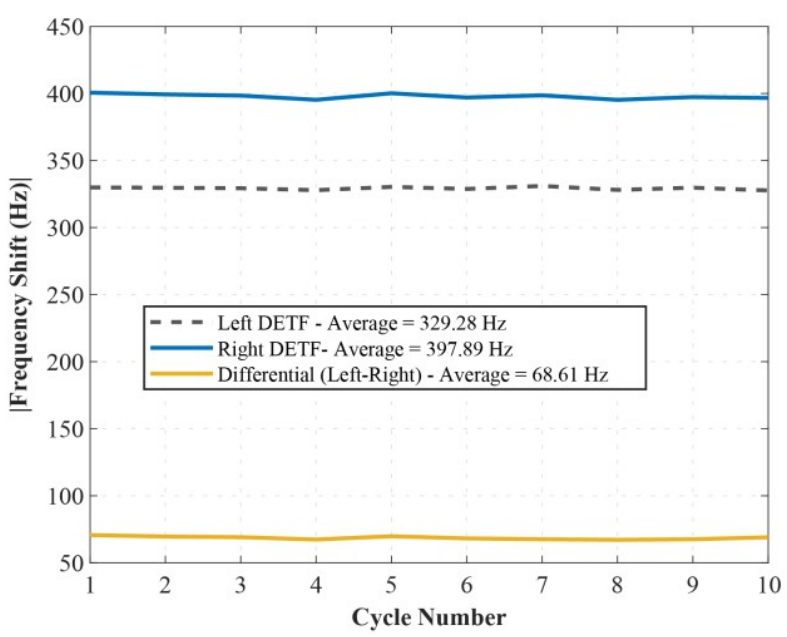

Fig. 6. Maximum frequency shift measured for a maximum force of about $15 \mathrm{~N}$, the test was repeated 10 times.

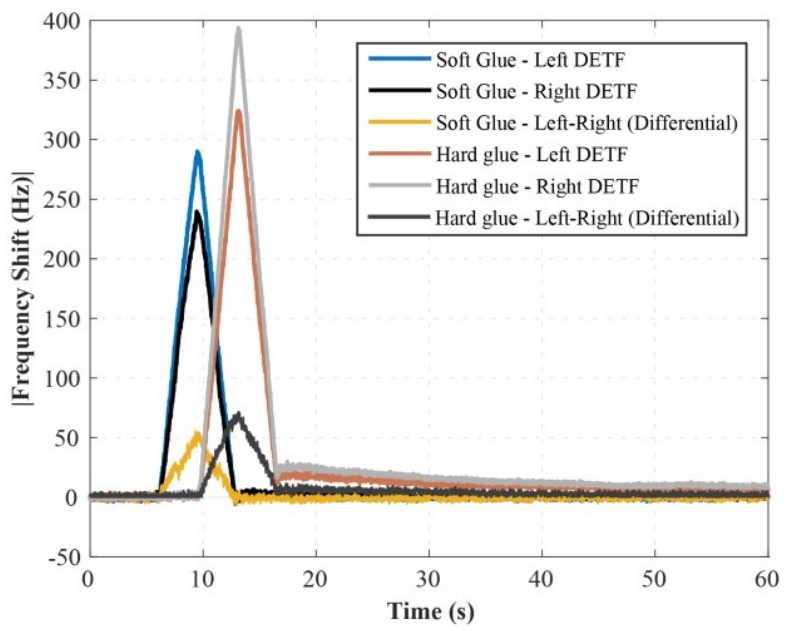

Fig. 7. Natural frequency drift of two devices glued with two different glues (hard and soft) for a maximum force of $15 \mathrm{~N}$.

$390 \mathrm{~Hz}$ and $289 \mathrm{~Hz}$, were measured on the left DETFs of the samples mounted on hard and soft glue, respectively. From this, one concludes, the glue with viscoelastic properties was able to reduce the influence of stress by approximately $26 \%$, considering only the measurement performed on the singleended DETFs. On the other hand, in what concerns the differential measurement, a maximum drift of $71.6 \mathrm{~Hz}$ (hardglue sample) and $55.5 \mathrm{~Hz}$ (soft-glue sample) were experimentally measured, representing a $22.5 \%$ reduction (lower than in single-ended measurement). This is explained by a higher stress non-uniformity on the sample mounted with soft-glue despite a lower nominal drift on the DETFs' response. From the experimental data analysis, the differential configuration has a higher influence on the reduction of stress sensitivity than the bonding glue.

Additionally, in Fig. 7 one can observe the sample mounted on hard-glue needs more than 30 seconds to return to the original response while the sample on soft-glue recovers instantaneously. Since the sample glued with epoxy is strongly attached to the substrate, the recovery of the different elements composing the setup, namely, the PCB or the chip carrier, is sensed by this sample. In other words, the device senses the movement of the different parts on the surroundings until the initial position is reached. This 
validates the PDMS-based glue as more effective to decouple stress, as expected.

\section{Long-term measurement}

Both samples were tested for a constant force applied over a time period of $100 \mathrm{~min}$. In Fig. 8, one observes the sample mounted on epoxy-based glue remains fairly stable while the differential response of the sample mounted on soft glue tends to the initial value. The response of this last is explained by the elastic properties of the soft glue since the stress relaxation and creep (mechanisms that reduce the stress applied to the sample) properties of viscoelastic materials appear when the material is held at a constant extension for some time, as observed in Fig. 8. The benefits of this glue are maximized for stimulus occurring over a long period, while for forces applied for few seconds (as shown in Fig. 7) the stress decoupling is lower. From this, one concludes the main advantage of the use of a soft glue to package resonant accelerometers is mainly related to longterm stimuli, while abrupt forces result in similar frequency shifts as devices strongly attached to the substrate.

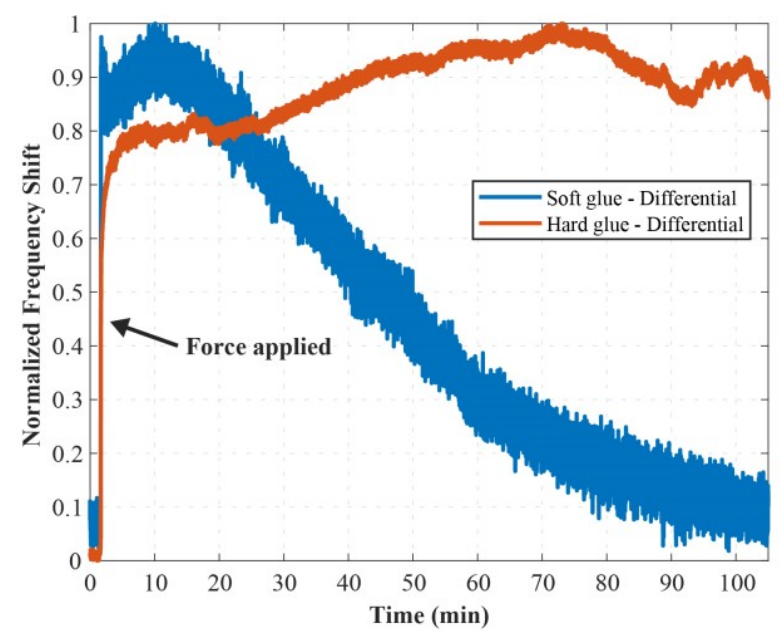

Fig. 8. Differential response of the sensors for a constant force applied for over 100 minutes.

\section{CONCLUSION}

A frequency modulated accelerometer based on doubleended tuning fork resonator, fabricated on a standard silicon micromachining process and encapsulated in vacuum was tested regarding the sensitivity to external forces applied directly on the chip carrier. Two techniques were used to minimize the drift of the sensor for stress, namely, a differential configuration and packaging using a soft PDMS based glue. The effectiveness of such approaches was experimentally measured, and the differential approach yielded a decrease on the stress sensitivity of approximately $81 \%$ (taking into account the value between the left DETF and the differential measurement on the sample glued with soft glue), being the most effective method for fast stimulus. The sample when packaged using soft glue demonstrated a drift $22.5 \%$ lower than using hard-glue, for the same force. The device response drift when glued with two materials with distinct properties was also shown along this work. The long-term response of the two samples when a constant force is applied for a time period above 100 min was measured, and the viscoelastic properties of the soft-glue (creeping and stress relaxation) resulted on a total recovery of the sensor response. This glue showed a great effectiveness on the longterm while the differential measurement was more adequate for fast changing stress. Although, the influence of stress was reduced using soft-glue and a differential measurement, these techniques are not enough to eliminate the dependency and more mechanisms, at the design level, should be implemented.

\section{ACKNOWLEDGMENT}

The authors would like to offer special thanks to the author Luis A. Rocha, who, although no longer with us, continues to inspire by his example and dedication to the students and collaborators he served over the course of his career.

The first author is supported by FCT- Fundação para a Ciência e Tecnologia through the grant $\mathrm{PDE} / \mathrm{BDE} / 114564 / 2016$. This work is supported by FCT with the reference project UID/EEA/04436/2019.

\section{REFERENCES}

[1] A. A. Seshia et al., "A vacuum packaged surface micromachined resonant accelerometer," J. Microelectromechanical Syst., vol. 11, no. 6, pp. 784-793, Dec. 2002.

[2] C. Comi, A. Corigliano, G. Langfelder, A. Longoni, A. Tocchio, and B. Simoni, "A Resonant Microaccelerometer With High Sensitivity Operating in an Oscillating Circuit," J. Microelectromechanical Syst., vol. 19, no. 5, pp. 1140-1152, Oct. 2010

[3] G. Laghi, C. R. Marra, P. Minotti, A. Tocchio, and G. Langfelder, "A 3-D Micromechanical Multi-Loop Magnetometer Driven OffResonance by an On-Chip Resonator," J. Microelectromechanical Syst., vol. 25, no. 4, pp. 637-651, Aug. 2016.

[4] Y. Yin, Z. Fang, Y. Liu, and F. Han, "Temperature-Insensitive Structure Design of Micromachined Resonant Accelerometers," Sensors, vol. 19, no. 7, p. 1544, Mar. 2019

[5] C. D. Do, A. Erbes, J. Yan, K. Soga, and A. A. Seshia, "Vacuum Packaged Low-Power Resonant MEMS Strain Sensor," J. Microelectromechanical Syst., vol. 25, no. 5, pp. 851-858, 2016.

[6] D. D. Shin, Y. Chen, I. B. Flader, and T. W. Kenny, "Epitaxially encapsulated resonant accelerometer with an on-chip micro-oven," in 2017 19th International Conference on Solid-State Sensors, Actuators and Microsystems (TRANSDUCERS), 2017, pp. 595-598.

[7] K. E. Wojciechowski, "Electronics for Resonant Sensors," University of California, Berkeley, 2005.

[8] E. E. Moreira, B. Kuhlmann, J. Gaspar, and L. A. Rocha, "Resonant Accelerometer based on Double-Ended Tuning Fork and a Force Amplification Mechanism," in Proceedings, 2018, vol. 2, no. 13, p. 1030.

[9] E. E. Moreira, B. Kuhlmann, J. Gaspar, and L. A. Rocha, "Small Size And Highly Sensitive Differential MEMS Accelerometer Based On Double-Ended Tuning Fork Resonators," in 20th International Conference on Solid-State Sensors, Actuators and Microsystems \& Eurosensors XXXIII (TRANSDUCERS \& EUROSENSORS XXXIII), 2019, vol. 3, no. June, pp. 602-605.

[10] Y. L. Chan and A. H. W. Ngan, "Invariant elastic modulus of viscoelastic materials measured by rate-jump tests," Polym. Test., vol. 29 , no. 5 , pp. 558-564, Aug. 2010 